\title{
A Decline in Response Variability Improves Neural Signal Detection during Auditory Task Performance
}

\author{
Gardiner von Trapp, ${ }^{1}{ }^{\circledR}$ Bradley N. Buran, ${ }^{1}$ Kamal Sen, ${ }^{4}{ }^{-}$Malcolm N. Semple, ${ }^{1,2}$ and Dan H. Sanes ${ }^{1,2,3}$ \\ ${ }^{1}$ Center for Neural Science, ${ }^{2}$ Department of Psychology, and ${ }^{3}$ Department of Biology, New York University, New York, New York 10003, and ${ }^{4}$ Department of \\ Biomedical Engineering, College of Engineering, Boston University, Boston, Massachusetts 02215
}

The detection of a sensory stimulus arises from a significant change in neural activity, but a sensory neuron's response is rarely identical to successive presentations of the same stimulus. Large trial-to-trial variability would limit the central nervous system's ability to reliably detect a stimulus, presumably affecting perceptual performance. However, if response variability were to decrease while firing rate remained constant, then neural sensitivity could improve. Here, we asked whether engagement in an auditory detection task can modulate response variability, thereby increasing neural sensitivity. We recorded telemetrically from the core auditory cortex of gerbils, both while they engaged in an amplitude-modulation detection task and while they sat quietly listening to the identical stimuli. Using a signal detection theory framework, we found that neural sensitivity was improved during task performance, and this improvement was closely associated with a decrease in response variability. Moreover, units with the greatest change in response variability had absolute neural thresholds most closely aligned with simultaneously measured perceptual thresholds. Our findings suggest that the limitations imposed by response variability diminish during task performance, thereby improving the sensitivity of neural encoding and potentially leading to better perceptual sensitivity.

Key words: amplitude modulation; attention; auditory cortex; neurometric; signal detection theory

\section{Significance Statement}

The detection of a sensory stimulus arises from a significant change in neural activity. However, trial-to-trial variability of the neural response may limit perceptual performance. If the neural response to a stimulus is quite variable, then the response on a given trial could be confused with the pattern of neural activity generated when the stimulus is absent. Therefore, a neural mechanism that served to reduce response variability would allow for better stimulus detection. By recording from the cortex of freely moving animals engaged in an auditory detection task, we found that variability of the neural response becomes smaller during task performance, thereby improving neural detection thresholds.

\section{Introduction}

The ability of a neuron to detect a sensory signal is generally thought to arise from a significant change in mean response magnitude with response variability as the principal limitation. While prior studies demonstrate that response magnitude changes when animals engage in a broad range of perceptual tasks (Hubel

Received April 19, 2016; revised Aug. 31, 2016; accepted Sept. 2, 2016.

Author contributions: G.v.T., B.N.B., M.N.S., and D.H.S. designed research; G.v.T. performed research; G.v.T. and K.S. contributed unpublished reagents/analytic tools; G.v.T. analyzed data; G.v.T., B.N.B., M.N.S., and D.H.S. wrote the paper.

This work was supported by National Institutes of Health Grants R01 DC014656 (D.H.S. and M.N.S.), T32 MH019524 (G.v.T.), and F31 DC013502 (G.v.T.). We thank Ishita Aloni, Mahfuzur Miah, Melissa Caras, Justin Yao, and Kristina Penikis for helpful discussions and editorial comments on the manuscript.

The authors declare no competing financial interests.

Correspondence should be addressed to Gardiner von Trapp, Center for Neural Science, New York University, 4 Washington Place, Room 809, New York, NY 10003. E-mail: g.vontrapp@nyu.edu.

DOI:10.1523/JNEUROSCI.1302-16.2016

Copyright $\odot 2016$ the authors $\quad 0270-6474 / 16 / 3611097-10 \$ 15.00 / 0$ et al., 1959; Spitzer et al., 1988; Burton et al., 1997; Treue and Maunsell, 1999; Reynolds et al., 2000; Steinmetz et al., 2000; Fritz et al., 2003, 2005; Chapman and Meftah, 2005; Elhilali et al., 2007; Schneider et al., 2014; McGinley et al., 2015a), response variability has received less attention because it is thought to scale reliably with firing rate. However, knowledge of response variance is essential to determining the sensitivity of neural signal detection. Therefore, we recorded from the auditory cortex of animals, as they performed a detection task, and tested the hypothesis that neural response variability can decrease during task performance, thereby increasing neural sensitivity and, potentially, perceptual sensitivity.

The most fundamental observation regarding the relation between variability and firing rate is that it scales with response magnitude in a Poisson-like manner (Tolhurst et al., 1983; Goris et al., 2014). This relation can be modulated by many factors, including anesthesia (Ter-Mikaelian et al., 2007; Ecker et al., 2014). Variability can even be affected by the onset of stimuli, a 
finding shown in many sensory areas and at different levels of sensory hierarchies (Churchland et al., 2010). More relevant to the present study, task engagement and attentional state are associated with a reduction in correlations between neuron pairs (McAdams and Maunsell, 1999; Reynolds et al., 2000; Cohen and Maunsell, 2009; Yoshida and Katz, 2011). Taken together, these studies establish that response variability is a dynamic property that may constrain or improve sensory encoding, thereby influencing perception.

To address whether response variability influences sensory encoding, we adopted a signal detection theory (SDT) framework, a model proven useful for establishing the relation between sensory encoding and perceptual abilities (Foley and Legge, 1981; Britten et al., 1992, 1996; Parker and Newsome, 1998; Gold and Shadlen, 2001; Luna et al., 2005). SDT posits that the central representation of a stimulus is described by a normally distributed neural signal (e.g., a range of firing rates). It follows that the ability to detect a stimulus is proportional to the difference between the variancenormalized means of a "signal present" and "signal absent" distribution. The SDT model would then lead to the prediction that stimulus detection sensitivity, or neural sensitivity, can improve as the variability of each distribution decreases.

To investigate whether response variability can influence neural sensitivity during listening, we recorded telemetrically from the core auditory cortex of gerbils both while they engaged in an amplitude modulation (AM) detection task and while they were disengaged from the task. AM is a favorable acoustic feature to examine because it is a component of natural sounds (Rosen, 1992; Singh and Theunissen, 2003; Elliott and Theunissen, 2009) and its encoding has been well characterized (Joris et al., 2004; Carney et al., 2013). Our findings support an encoding framework in which neural variability diminishes during task performance, leveraging greater neural sensitivity and potentially greater perceptual sensitivity.

\section{Materials and Methods}

\section{Subjects}

Four adult gerbils (Meriones unguiculatus, three males) were weaned from commercial breeding pairs (Charles River). All procedures related to the maintenance and uses of animals were approved by New York University's Institutional Animal Care and Use Committee.

\section{Behavior}

Stimuli

Gerbils were placed in a plastic cage in a sound-isolation booth (GretchKen Industries) and observed via a closed-circuit monitor. A personal computer, connected to a digital input/output interface (TDT RZ6, Tucker-Davis Technologies) controlled the timing of the acoustic stimuli, reward delivery, and acquisition of behavioral and electrophysiological data. AM stimuli were generated by a Tucker-Davis Technologies system (RZ6) and delivered via a calibrated tweeter (DX25TG05-04,
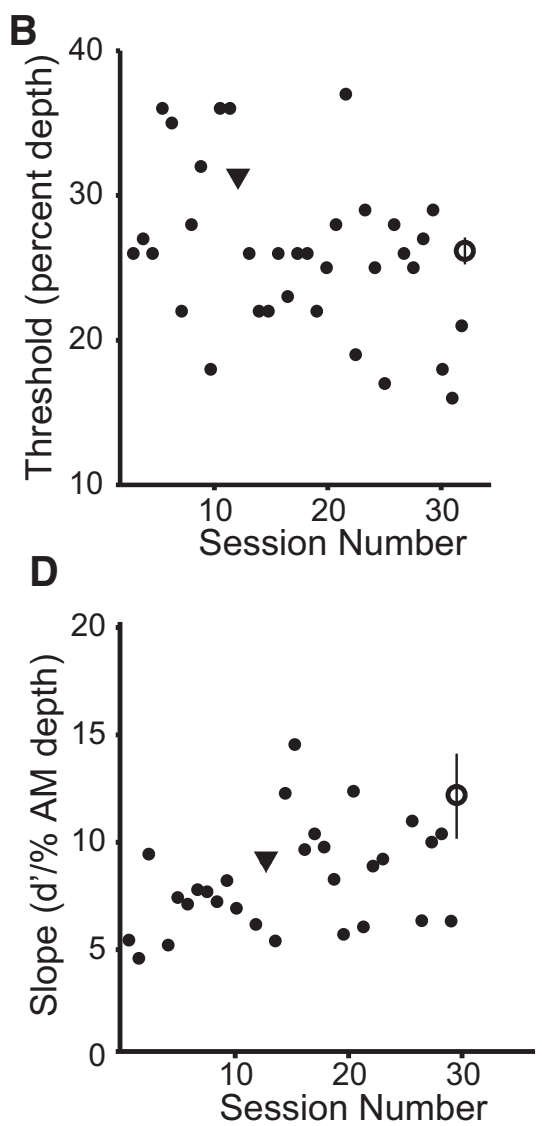

Figure 1. Behavioral performance on an AM detection task. $\boldsymbol{A}$, Exemplar fit psychometric function obtained for one gerbil during one session. Black dashed line indicates detection threshold $\left(\boldsymbol{d}^{\prime}=1\right)$. $\boldsymbol{B}-\boldsymbol{D}$, Individual (small black circles) and average (large open circles) psychometric thresholds, psychometric function slopes, and false alarm (FA) rates are plotted. Values measured from exemplar function are indicated by black triangles. Error bars represent SEM.

Vifa) positioned $1 \mathrm{~m}$ above the center of the test cage. The gerbil's distance from the speaker could only vary maximally by $\approx 0.6 \mathrm{~cm}$ (or $\approx 10$ $\mathrm{cm}$ in the vertical or horizontal plane) with respect to the center of the cage. Sound level was measured with a spectrum analyzer (3550, Bruel \& Kjaer) via a one-quarter inch free-field condenser microphone positioned at the head location when aligned with the nose port. Sound level remained constant ( $50 \mathrm{~dB}$ equivalent SPL) throughout the trial to exclude the use of energy as a cue. The carrier was broadband noise, with a $25 \mathrm{~dB}$ roll-off at $3.5 \mathrm{kHz}$ and a $25 \mathrm{~dB}$ roll-off at $20 \mathrm{kHz}$. All stimuli had a $200 \mathrm{~ms}$ ramp, followed by an unmodulated period of $200 \mathrm{~ms}$, and then transitioned to an amplitude modulated or unmodulated signal. The delay period prevented animals from making a decision at stimulus onset, and yielded a similar onset response from auditory cortex neurons for both Go and Nogo stimuli.

\section{Training}

Animals were placed on controlled water access, and trained to discriminate between an unmodulated and $5 \mathrm{~Hz}$ sinusoidally AM frozen broadband noise using a positive-reinforcement procedure (Buran et al., 2014b; Sarro et al., 2015). Each animal was initially trained to begin a trial by poking its nose into a cylindrical port, and to approach a water spout upon presentation of an AM stimulus (i.e., the Go signal). Water reward $(20 \mu \mathrm{l})$ was delivered via a syringe pump (NE-1000, New Era). After animals learned to initiate Go trials, they were trained to repoke upon presentation of an unmodulated noise (i.e., the Nogo signal).

\section{Testing}

Perceptual ability was assessed by presenting Go trials at five different AM depths that bracketed the animal's psychometric threshold. Unmodulated Nogo trials were interleaved with Go trials, and randomly 
A

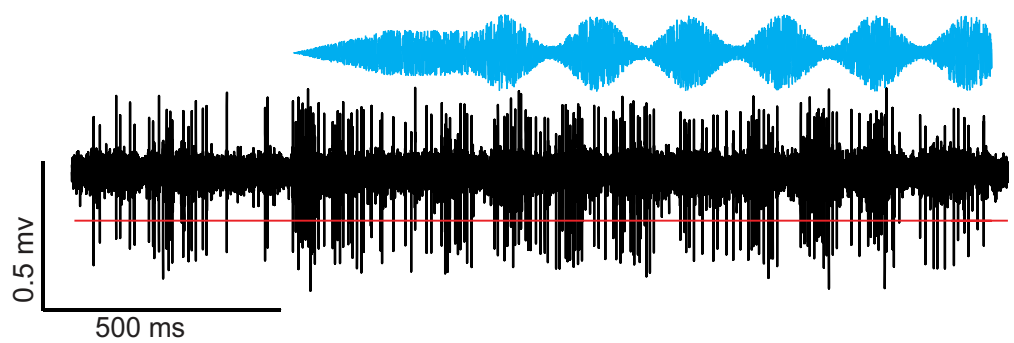

B

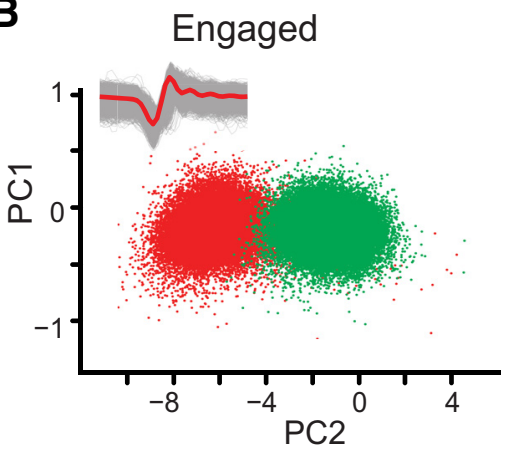

C
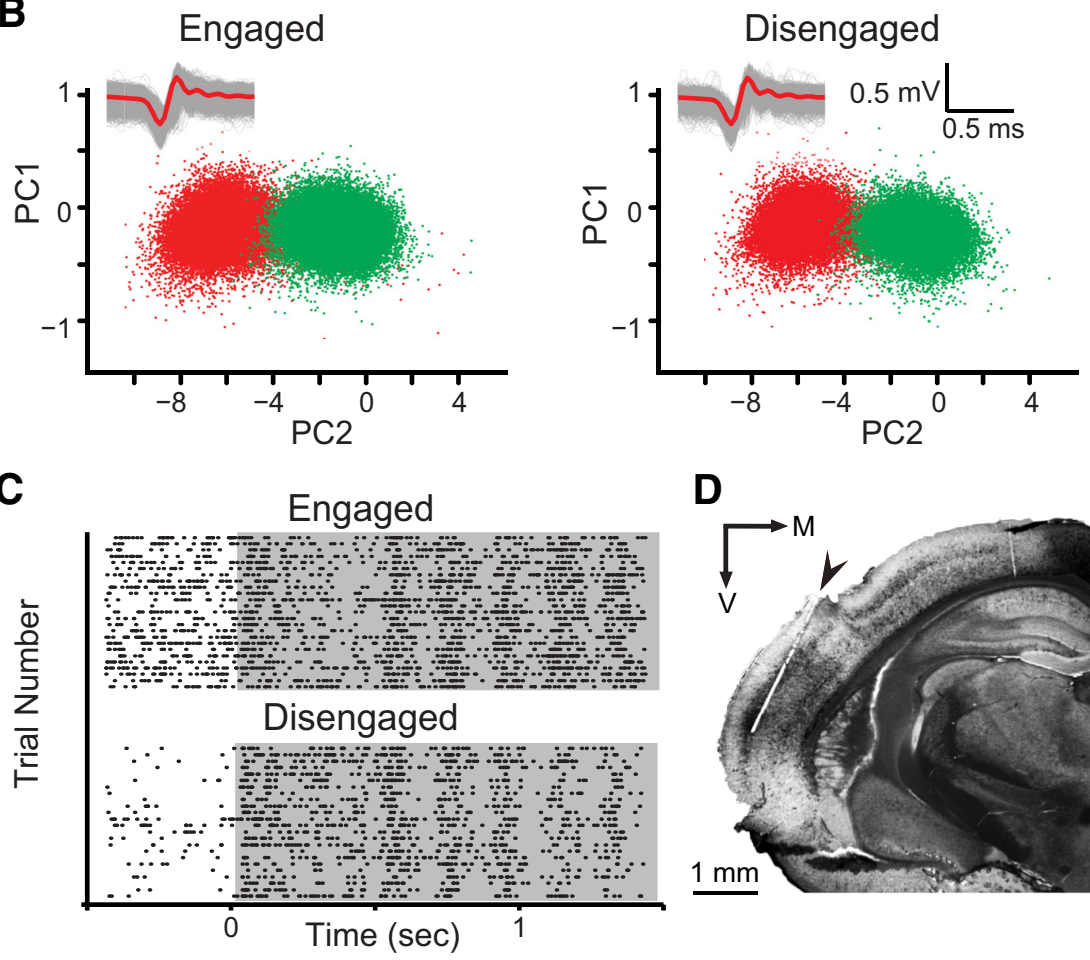

D

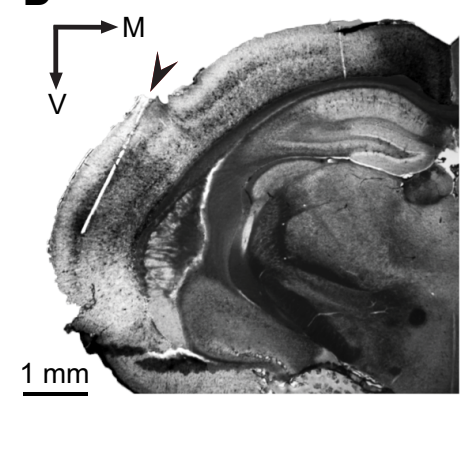

Figure 2. Selection of candidate waveforms for neurometric analyses. $A$, Raw waveform of a neural response to AM noise (blue envelope) during task engagement. Candidate waveforms were $>4$ SDs above noise floor (red line). B, PCA plot in which two waveform clusters are separated (red and green), both while an animal is engaged (left) or disengaged (right). Insets show raw waveforms (gray lines) and averages (red line) for red cluster. $\boldsymbol{C}$, Example raster for unit shown in $\boldsymbol{B}$. Gray shaded area indicates period when stimulus was present. $\boldsymbol{D}$, Coronal section shows an electrode track (arrow), with staining for wisteria floribunda agglutinin to reveal the primary auditory cortex.

presented with equal probability. In the engaged condition, animals were required to withhold from responding for a $\geq 1 \mathrm{~s}$. In trials scored as a hit (correctly approaching the water spout on a Go trial) animals were rewarded. For false-alarm (FA) responses (incorrectly approaching the water spout on a Nogo trial) a $2-8 \mathrm{~s}$ timeout period was initiated during which the room light was switched off and animals were unable to initiate a trial.

In an experiment separate from measuring psychometric functions, we shifted the decision criterion within a session. For this experiment, we used two of the four animals and a method previously applied in human studies (Green and Swets, 1966). First, full psychometric functions were collected and the average threshold across the three sessions was calculated. Criterion was subsequently shifted by first presenting Go stimuli with a $45 \%$ probability, and then changing to $80 \%$ probability halfway through the testing session. During these experiments, Go stimuli were presented at the predetermined detection threshold value, along with a suprathreshold value (i.e., 100\% depth).

\section{Psychometric analysis}

Psychometric sensitivity was determined by fitting the percentage of Go responses, plotted as a function of AM depth, using a maximum likelihood procedure from the open-source package Psignifit for Matlab. Sig- nificant fits for all behavior sessions were obtained using a linear transform of stimulus intensity values (i.e., the mw0.1 core available in the bootstrap inference algorithm) fitted by a normal cumulative distribution function. The function is parameterized as follows:

$\Psi(x ; m, w, y, \lambda)=\gamma+(1-\gamma-\lambda) ;$

$F(x ; m, w)$

where $F(x ; m, w)=\Phi\left(\frac{z(\delta)}{w}(x-m)\right)$; $\mathrm{z}(\delta)=\Phi^{-1}(1-\delta)-\Phi^{-1}(\delta)$ and where $\phi$ is the inverse of the cumulative Gaussian, $x$ represents stimulus difficulty (AM depth), $m$ is the midpoint, $w$ is the width of the interval over which $F(x ; m ; w)$ rises from $\delta$ to $1-\delta, \lambda$ is the lapse rate, and $\gamma$ is the FA rate. Both $m$ and $w$ were unconstrained and $\delta$ was fixed at 0.1 (the default value set by Psignifit). Prior distributions for FA rate and lapse rate are identical to previous studies in the laboratory (Buran et al., 2014a; Caras and Sanes, 2015) and were determined according to guidelines described previously (Frund et al., 2011).

From the fitted percentage Go response functions, a $d^{\prime}$ function was calculated with $d^{\prime}$ defined as $z$ (hit fraction $)-z$ (FA fraction). For the purposes of this study, we define psychometric threshold as the AM depth at which the fitted $d^{\prime}$ function $=1$. To validate the fits of the actual data, 2000 datasets were generated using a bootstrapping procedure, and fits were calculated. For each fit, a deviance, a measure describing the goodness of fit, was calculated. Fits generated from actual data were not considered valid if their measured deviance was not within the 95th percentile of the deviance measured from the simulated datasets. Lapse rate and FA rate were determined by finding the minimum and maximum value of the fit percentage Go response function, respectively. Slope was determined by finding the slope of the fit psychometric function at $d^{\prime}=1$.

The analyses were performed on all behavior sessions in which the FA rate was $\leq 30 \%$, and the animal performed $\geq 200$ trials. The average number of behavioral trials was $305 \pm 54$ trials per session (mean \pm SEM; $\sim 150$ Nogo trials, and 150 Go trials with 20 trials at each AM depth). Of the 40 sessions where physiology data were collected, five failed to meet acceptance criterion and were thus not included in the analysis.

\section{Electrophysiology}

Electrode implantation

After an initial set of psychometric functions was obtained (Fig. 1), a 16 -channel silicone probe with recording contacts evenly spaced across a $600 \times 600 \mu \mathrm{m}$ grid was implanted in the left core auditory cortex (Electrode model A4x4-4mm-200-200-1250-H16_21 mm, Neuronexus). The electrode array was inserted at a $25^{\circ}$ angle such that the contacts were generally located within the same cortical layer (Fig. $2 D$, electrode track), and response latencies and tonotopic gradients were consistent with core auditory cortex characteristics observed previously (Ter-Mikaelian et al., 2007; Rosen et al., 2010; Buran et al., 2014b). At the termination of each experiment, animals were perfused with a fixative, and the brains were extracted and sectioned. In selected sections, the recorded area was visualized using an immunohistochemical procedure that selectively labels layers IV and VI in the core auditory cortex (Brückner et al., 1994). Alternate sections were visualized with a Nissl stain. The histological data 

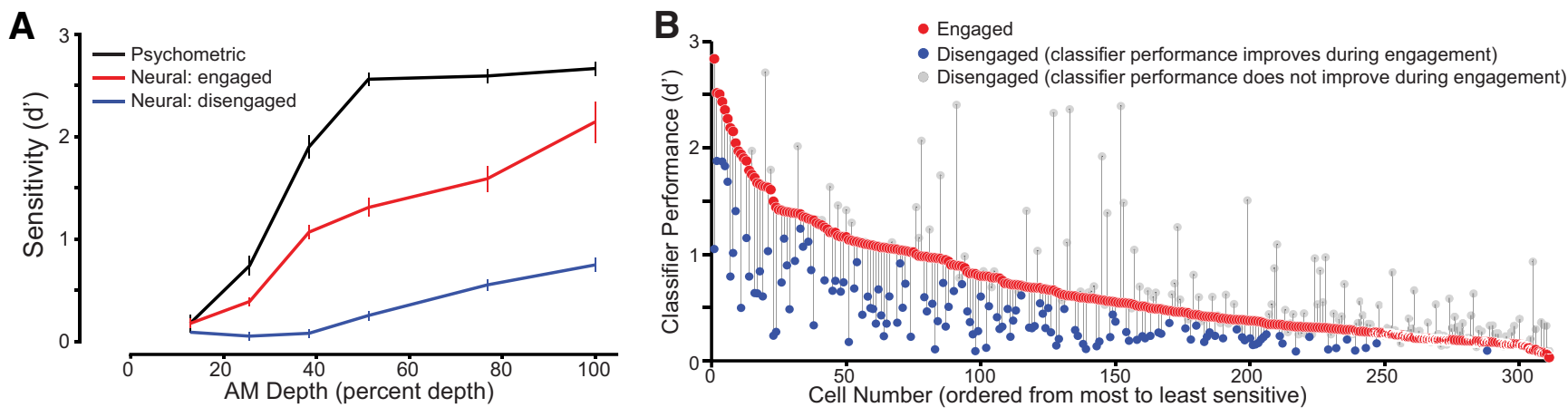

Figure 3. Population comparison of neurometric sensitivity while animals were engaged or disengaged in the AM detection task. $A$, Perceptual sensitivity (black) and neural sensitivity during the engaged (red) and disengaged conditions (blue). There was a significant difference between engaged and disengaged firing rate-based neurometric functions at AM depths $>12.8 \%$. $\boldsymbol{B}$, Task engagement led to an improvement in classifier performance for a subset of units. Pattern classifier analysis revealed that neurometric sensitivity to the AM stimulus was altered between engaged and disengaged states. The change in classifier performance for individual units is illustrated by the thin gray lines connecting classifier performance during the engaged (red circle) and disengaged conditions (blue circles, units for which performance improved; gray circles, units for which performance declined or did not improve). Units are ordered by classifier performance during the engaged state, from best to worst. Error bars represent SEM.

confirmed that the electrodes passed through the auditory cortex, and the electrode contacts were largely constrained to layers III or IV for each gerbil used in this study (Fig. 2D).

\section{Data acquisition}

Physiology and psychometric data were acquired concurrently while animals were engaged in the AM detection task using a telemetric headstage and receiver (W16, Triangle Biosystems). Engaged behavioral sessions lasted between 40 and $60 \mathrm{~min}$ and disengaged 30-40 min. The neurophysiological data were recorded via a preamplifier and analog-to-digital converter (TB32, Tucker-Davis Technologies).

Neurophysiological data were also acquired 5-10 min after task performance when animals were disengaged. Following implantation, gerbils were trained to assume a fixed position and remain still but alert in the testing cage. Trials were not presented unless gerbils were stationary. Gerbils' posture and eyes were monitored via closed circuit television to assure wakefulness and stillness. To indicate the start of the disengaged condition, the water spout and nose port were removed from the cage, and the direction of the room lighting was changed from a light source positioned on the floor (engaged condition) to an overhead light (disengaged condition). Stimuli were identical to the engaged condition, and were presented with an interstimulus interval (ISI) that varied randomly between 1 and $1.75 \mathrm{~s}$. The disengaged ISI was chosen to approximate the range of response times of animals observed during behavior sessions. By using this procedure, we sought to minimize the changes in animal position with respect to stimulus while retaining the naturalistic advantages of a freely moving preparation. Furthermore, the overhead speaker minimized interaural cues as well as differences in distance from the speaker. Finally AM stimuli are robust to changes in carrier level up to $\sim 15 \mathrm{~dB}$ (Malone et al., 2007), far greater than variations occurring in the present experiment.

Each recording channel was referenced to an across-channel average (Ludwig et al., 2009) and high-pass filtered at $300 \mathrm{~Hz}$. For individual channels, the noise floor was calculated (Quiroga et al., 2004) and candidate waveforms that exceeded 4 SDs from the noise floor were selected. Candidate waveforms were then sorted using a principal components analysis (PCA)-based clustering algorithm (KlustaKwik) and isolation was assessed using quality metrics (Fee et al., 1996; Hill et al., 2011). Extracted action potentials were further sorted using PCA clustering, as described in Materials and Methods (Fig. 2B). Sorting was done separately for engaged and disengaged conditions. To confirm that recordings were stable across behavioral conditions, PCA plots and waveforms were compared between the engaged and disengaged conditions. Units that had significantly nonoverlapping PCA or waveforms were rejected from the analysis.

Our recordings included well isolated single units that demonstrated clear separation in PCA space, with $<10 \%$ of spikes violating the default refractory period of $2.5 \mathrm{~ms}$ (Fee et al., 1996; Hill et al., 2011). However, the majority of recording sites contained spikes from several unresolved units and thus did not meet this criterion. Separate analyses of these two populations revealed no systematic differences; we therefore combined the populations for all analyses reported here. We also found no between-animal differences in basic response properties (firing rate, etc.). The quality of data was similar across animals (e.g., signal-to-noise ratio, and ratio of multiple units to single units per animal). However, the number of units obtained from one animal (nine units) was far fewer than that obtained from the remaining animals $(\approx 100$ units per animal). Given that we had no reason to reject these units, they were included in the study.

\section{Neurometric analysis}

Each unit's firing rate was calculated from a $1 \mathrm{~s}$ period of the spike train corresponding to the initial modulation of the stimulus. Since there is evidence that AM is represented by both temporal and rate codes (Liang et al., 2002; Joris et al., 2004; Bartlett and Wang, 2007; Malone et al., 2007; Wang et al., 2007, 2008; Rosen et al., 2010, 2012; Yin et al., 2011; Johnson et al., 2012; Carney et al., 2013), we performed analyses that addressed changes in both rate and temporal codes. Here we only show data examining the rate code because we did not observe a behaviorally gated improvement in neural sensitivity for a temporal code. This observation is consistent with a recent awake-behaving primate study employing an AM depth detection task (Niwa et al., 2012).

The $1 \mathrm{~s}$ duration used for calculating firing rate was chosen based on the average behavioral response time of animals to Go trials (1.6 \pm $0.6 \mathrm{~s}$; with minimum response time being $1 \mathrm{~s}$; see Testing). A neurometric firing rate-based $d^{\prime}$ was calculated at each Go value (AM depth) by normalizing the firing rate by an SD pooled across all stimuli ( $Z$ score), and subtracting the Nogo value (unmodulated signal) from each subsequent Go stimulus; thus: $d_{\text {FIRING RATE }}^{\prime}=z(\mathrm{AM}$ $\left.\operatorname{depth}_{G o}\right)-z\left(\right.$ unmodulated $\left._{\text {Nogo }}\right)$.

To compensate for differences in discharge magnitude for individual units across AM depths, we also calculated a baseline-corrected firing rate. For each unit, an average firing rate for the unmodulated stimulus was calculated, and then subtracted from firing rates on all trials. The result was an adjusted firing rate that reflects increases in discharge rate beginning at zero value for all units.

To calculate neurometric thresholds, firing rate-based $d^{\prime}$ versus AM depth functions were fit to a sigmoid using nonlinear regression. A best fit was produced by iteratively calculating the residual sum of squares of estimated fits until the error term was minimized (Matlab function nlinfit; Mathworks). Fitting was performed individually for each unit. Goodness of fit was then assessed by calculating the significance of the Pearson's correlation coefficient. Threshold was defined as the AM depth at which the fitted neurometric $d^{\prime}$ function $=1$. A sigmoid was chosen as a close approximation for the normal cumulative distribution used to fit psychometric functions. 


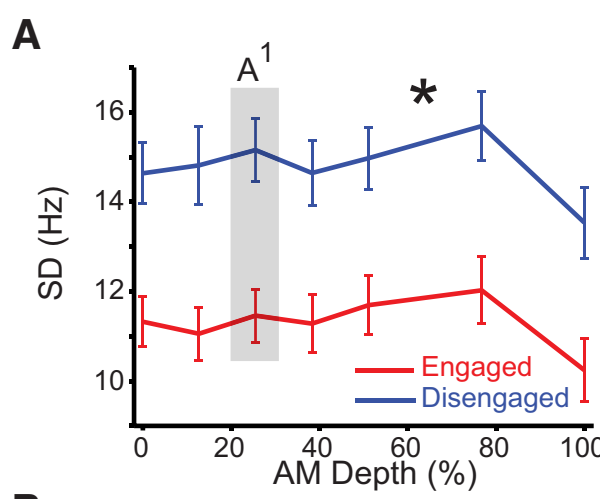

B
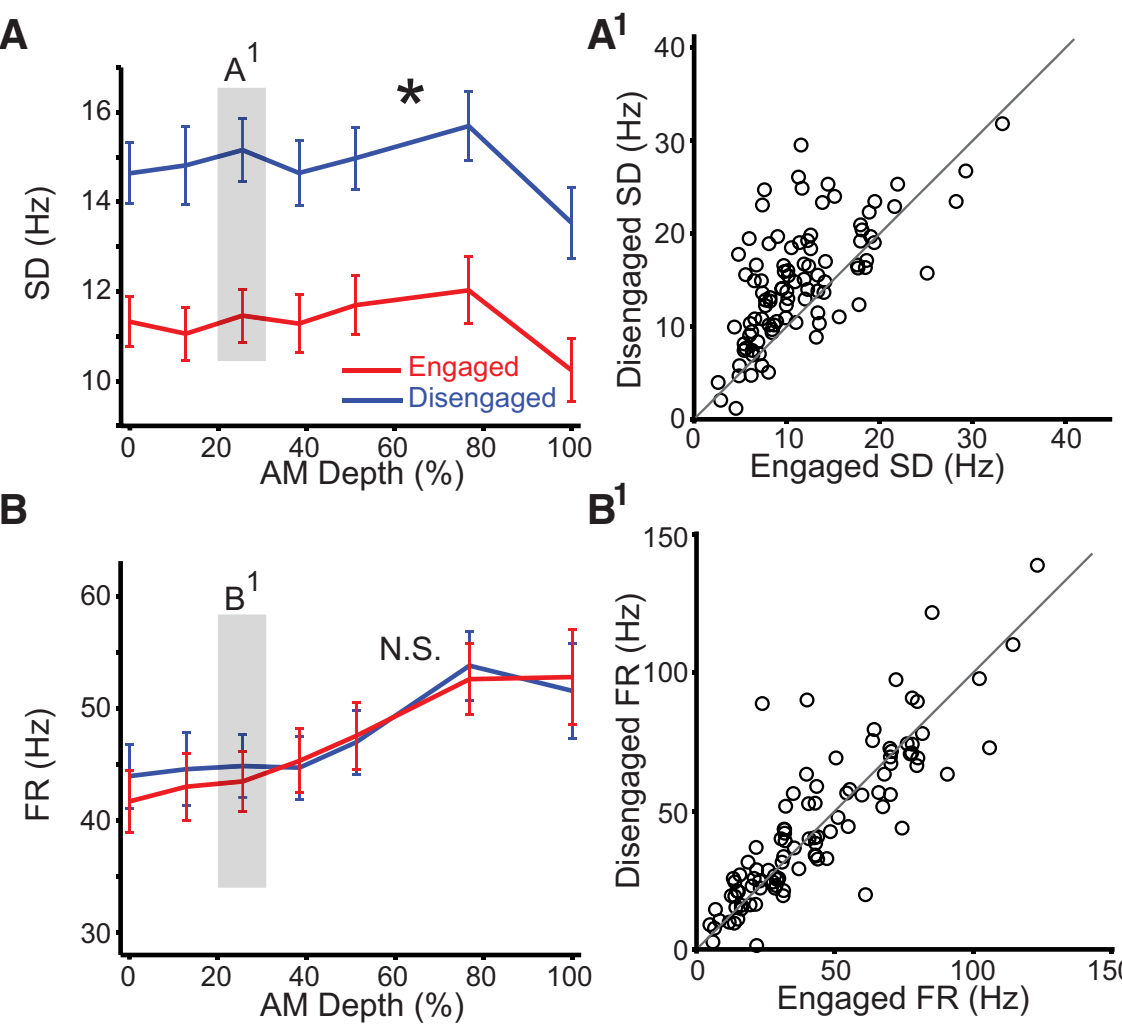

$B^{1}$

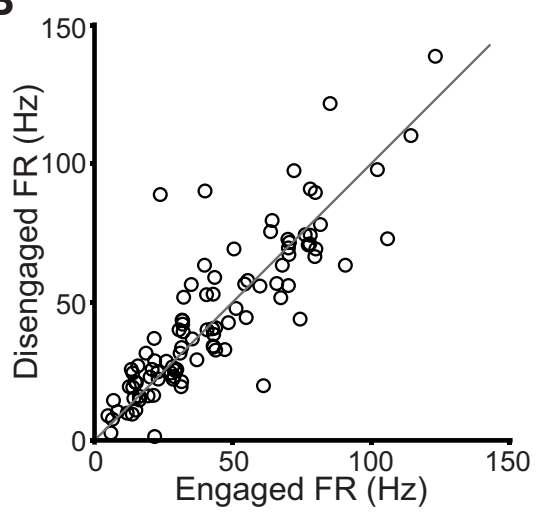

C

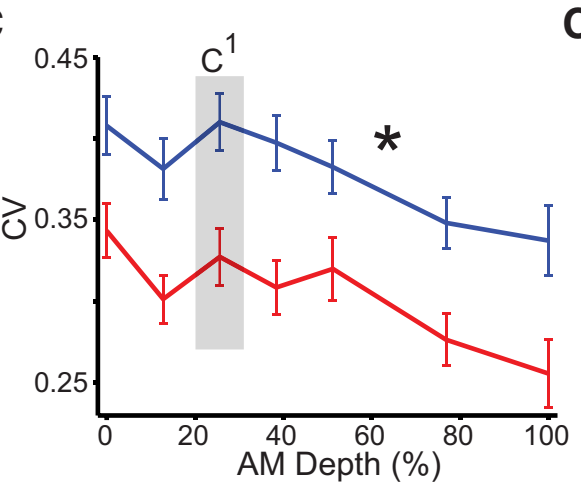

$\mathrm{C}^{1}$

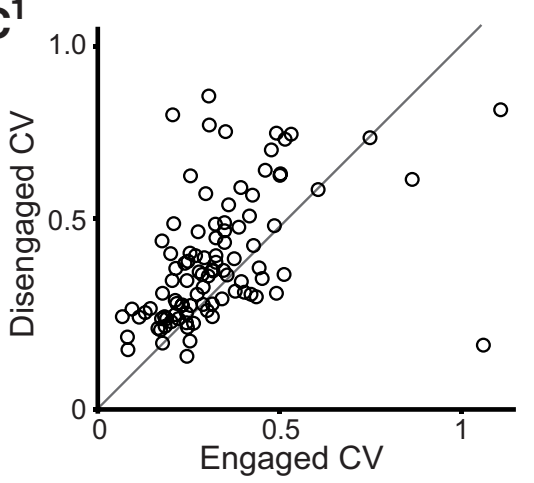

Figure 4. The increased neural sensitivity and classifier performance is driven by a decrease in response variability. $\boldsymbol{A}-\boldsymbol{C}$, Plots of the change in standard deviation (SD), firing rate (FR), and coefficient of variation (CV) for units that displayed the greatest improvement in classifier performance from the disengaged to engaged conditions (Fig. 3B). There was a significant reduction in SD and CV during task performance (asterisk, see text for statistical values). $\boldsymbol{A}^{\mathbf{1}}-\boldsymbol{C}^{\mathbf{1}}$, Scatter plots of individual units (black circles) from an AM depth near psychometric threshold (26\%), indicated by the shaded box in $\boldsymbol{A}-\boldsymbol{C}$. Error bars represent SEM.

\section{Pattern classifier}

To identify and classify units using a performance metric based on neural responses from trial to trial, we adopted a pattern classifier analysis (Machens et al., 2003; Narayan et al., 2006; Wang et al., 2007; Billimoria et al., 2008; Schneider and Woolley, 2010). The pattern classifier produces a performance metric that is highly dependent on the reliability of a unit's response from trial to trial, thus allowing for the identification, comparison, and quantification of units with highly consistent trial-by-trial responses. While the purpose of the classifier was solely to parse units into groups, we optimized the classifier parameters to obtain a best performance from each unit.

Template matching. First, for an individual unit, two templates were selected: a single spike train from a Go trial, and one from a Nogo trial. Using firing rate as the metric, subsequent spike trains for this particular set of Go and Nogo values were then selected without replacement, and assigned to the Go or Nogo template based on the smallest difference in firing rate between template and trial. Since template trials were selected randomly, spike train classification was repeated 1000 times with a different Go/Nogo template each time to remove template selection bias.

Scoring classifier performance. From this comparison, a percentage correct assignment score of a trial to a template was generated. Classification of a trial was scored as follows: if a Go trial was assigned to a Go template, then it was scored as a hit; if a Go trial was assigned to the Nogo template, then it was scored as an FA. The percentage hit and FA scores were averaged across repetitions and then normalized $(Z$ score) and converted to a classifier-based $d^{\prime}$, as described above. This analysis was repeated for the same units that were recorded during the period when animals were disengaged from the task.

Parsing units. Units were ranked by maximum classifier-based $d^{\prime}$ measured during task performance and separated into groups based on their change in classifier-based $d^{\prime}$ across the engaged and disengaged conditions. Maximum classifier-based $d^{\prime}$ is the maximum classifier $d^{\prime}$ value a unit could produce for any stimulus or decay constant $(\tau)$ value (see details below for $\tau$ values). Units that displayed a $\geq 0.2$ increase in classifier $d^{\prime}$ from the engaged to the disengaged condition were considered to have increased in classifier performance from one condition to the other. Units that displayed a decrease in classifier $d^{\prime}$ were considered to have decreased in performance, and units displayed a change $\leq 0.2$ were considered to have no change in performance. The criterion of 0.2 change in classifier $d^{\prime}$ performance represents a change that falls beyond the $95 \%$ confidence interval of the distribution of changes in classifier performance. We also verified that the set of increased and decreased values is significantly different from a zero mean distribution representing no change in classifier performance (onesample $t$ test, $p<0.001$ ).

Relevance of chosen classifier metrics. Given that there are several potential ways in which AM may be encoded, the classifier analysis was performed using three different methods. In addition to firing rate, we used the van Rossum (VR) metric. The VR metric uses the Euclidean distance between two spike trains to quantify a measure of dissimilarity (van Rossum, 2001). The spike trains were then classified using a "nearest-neighbor" classifier based on the spike distance. The final method also used the VR metric, but instead of the nearest-neighbor classifier, it used a K-means classifier, which optimized spike train discrimination by using an iterative clustering algorithm (Duda et al., 2001; Schneider and Woolley, 2010). For the K-means classifier, the initial clusters were based on the selected Go and Nogo template. All metrics used $1 \mathrm{~s}$ of the spike train, and were sampled at a frequency of $1000 \mathrm{~Hz}$ beginning at the time when the AM depth was modulated. For VR and K-means, each spike train was convolved with an exponential function with a given decay constant. The nine different decay constants used were as follows: $2,4,8,16,32,64,128,256$, and $512 \mathrm{~ms}$.

\section{Results}

Psychometric performance: sensitivity to AM depth

To record responses from neurons in an animal engaged in a perceptually demanding auditory-guided behavior, animals were 
trained to perform a Go-Nogo AM detection task. Before implantation, animals were trained until they reached asymptotic performance. After implantation, psychometric and physiology data were collected concurrently from three animals. AM detection thresholds $\left(d^{\prime}=1\right)$ were similar across animals (Fig. 1A). Average AM threshold across all sessions was $26 \pm 1 \%$ AM depth (or $-13.1 \pm 0.4 \mathrm{~dB}$ relative to $100 \%$ depth; Fig. $1 B$ ), which is consistent with detection thresholds reported for other species (O'Connor et al., 2011; Carney et al., 2013). No betweenanimal differences were observed for AM threshold, FA rate $(22 \pm 1 \%$; Fig. $1 C)$, psychometric slope determined at $d^{\prime}=1$ (12.2 $\pm 2.0 d^{\prime} /$ percentage AM depth; Fig. $1 D)$, or lapse rate $(4.6 \pm 0.42 \%$; data not shown). Presurgery psychometric performance was compared with postsurgery performance. Before implantation, animals displayed psychometric thresholds of $26 \pm 2 \%$ AM depth, FA rates of $20 \pm 1 \%$, slopes of $12.9 \pm 2.0\left(d^{\prime} / \mathrm{AM}\right.$ depth percentage), and lapse rates of $3.4 \pm 0.3 \%$ (data not shown). Statistical comparisons of psychometric performance before and after implantation did not reach significance.

\section{Neural sensitivity and classifier-based performance improves during task engagement}

A primary objective of this study was to compare sensitivity of neural responses to identical AM depth stimuli during two behavioral states: engaged in a perceptually demanding auditory task or disengaged (assuming a fixed position in the test cage; see Materials and Methods). Raw physiology (Fig. 2A) data were preprocessed before extraction of candidate waveforms. Extracted action potentials were further sorted using PCA clustering, as described in Materials and Methods (Fig. 2B). To confirm that recordings were stable across behavioral conditions, PCA plots and waveforms were compared between the engaged and disengaged conditions. Abnormal waveforms were rejected and clusters were classified as "single" or "multiple" units based on quality metrics (ISI and autocorrelation plots). Rasters (Fig. 2C) were also examined, and electrode tracks were localized to layer III or IV of the primary auditory cortex (Fig. $2 D$ ). Of the total 311 recordings, $16 \%$ were considered single units. Data from single and multiple units were analyzed separately; results were consistent across single and multiple unit populations, and were therefore combined.

To assess changes in average neural sensitivity from an engaged to a disengaged state, a firing rate-based neural $d^{\prime}\left(d_{F R}^{\prime}\right)$ was calculated for each unit. Neurometric functions were obtained for each unit by plotting $d_{F R}^{\prime}$ at each tested AM depth (see Materials and Methods). Neurometric sensitivity was measured during the engaged condition (when gerbils performed the auditory task) and disengaged condition (assuming a fixed position in the test cage). In Figure $3 A$, the average engaged and disengaged neurometric sensitivity is plotted together with the average psy- chometric performance. Units were more sensitive to AM depth when animals were engaged in a task than when they were disengaged. Neurometric sensitivity was greater for AM depths near or above animals psychometric threshold [e.g., $>12.8 \%$; paired $t$ test with Bonferroni's adjusted $\alpha$ levels of $p=0.0014(0.01 / 7)$, $p<0.0014$.

Given the average increase in neural sensitivity, we sought to rank and parse out individual units based on similarity of responses across trials. Therefore we chose a pattern classifier scheme that calculates a performance metric for each unit that is highly dependent on the similarity of neural responses from trial to trial. From each unit's classifier results, we constructed a classifier performance function: classifier $d^{\prime}$ as a function of AM depth. We then extracted each unit's best performance (maximum classifier $d^{\prime}$ ) for each of the 311 units. In addition to firing rate, a K-means and spike-timing (van Rossum, 2001) metric were also used to quantify neural responses and determine differences between the two behavior conditions.

To compare each unit's classifier performance in the engaged and disengaged states, best classifier $d^{\prime}$ was derived from the classifier neurometric functions. We ranked and plotted each unit's classifier performance during task engagement from highest to lowest along with unit's corresponding classifier performance in the disengaged condition (Fig. 3B). During task engagement, $32 \%$ of units improved in performance (red circles with blue 
A
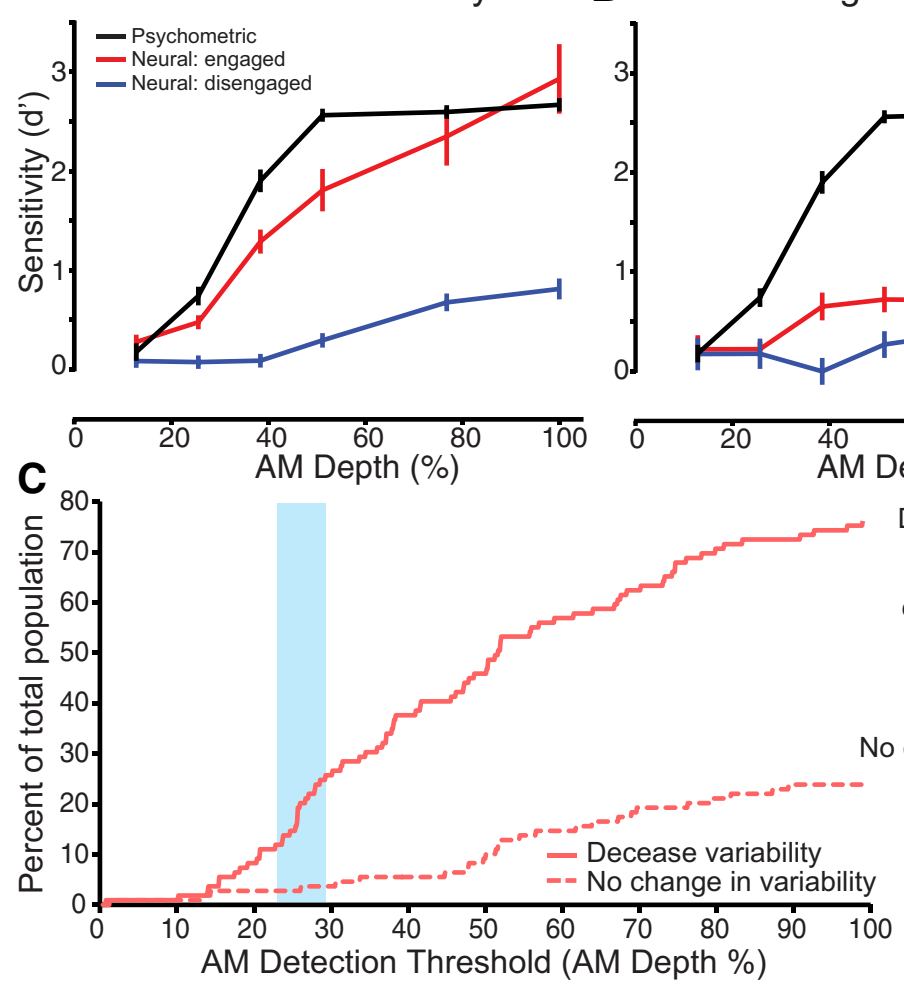

B No change in variability

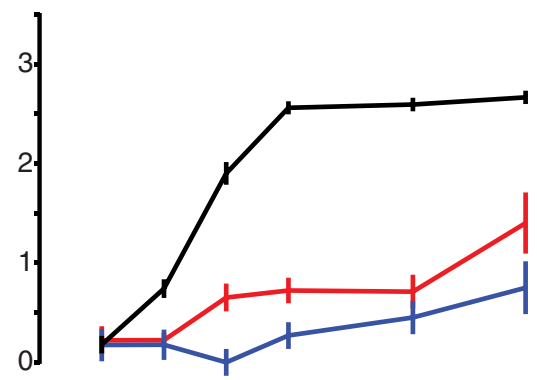

Figure 6. Direct comparison of neurometric and psychometric sensitivity for units that decreased response variability and units that did not decrease response variability during task engagement. $\boldsymbol{A}$, Psychometric sensitivity (black) was closer to neural sensitivity during task engagement (red) than when disengaged (blue) for units that decrease in response variability. $\boldsymbol{B}$, Neural sensitivity during task engagement (red) was not as well matched to behavior (black line) for units that did not change in variability. C, Units that decreased response variability (dashed red line) from the disengaged to engaged conditions exhibited neurometric thresholds that more closely approximated psychometric thresholds (blue rectangle). Insets (on right) show example neurometric functions (light red points) and fits for one example unit that displayed decreased variability (top right; solid light-red line) and one that displayed no change in variability (bottom right; dashed light-red line) from the disengaged to engaged conditions. Error bars represent SEM.

points below). Of the remaining units, $15 \%$ declined in performance and 27\% displayed no change (gray points), while $26 \%$ did not reach a performance of $d^{\prime}>0$ at any depth. We also compared the average classifier performance for the engaged $(0.68 \pm 0.03)$ and disengaged $(0.58 \pm 0.03)$ condition, and found significantly better classifier performance from units during task performance (paired $t$ test, $p=0.002$; data not shown). Thus, while changes in classifier performance were heterogeneous, task engagement led to a net improvement in classifier performance despite reducing the performance of a subpopulation. Classifier results were consistent regardless of which neurometric (i.e., VR or K-means) was used by the classifier.

\section{Increased neural sensitivity is driven by a decrease in variability}

The classifier analysis revealed unit subpopulations for which classifier performance increased or decreased during task engagement. Therefore, we asked whether firing rate properties could account for the difference between these two subpopulations. For units in which classifier performance improved during task engagement (Fig. 3B, blue circles connected to red circles), the SD of response firing rate was significantly lower during task engagement (Fig. $4 A$ ). Figure $4 A^{1}$ shows a scatter plot comparing the response variability during the engaged versus disengaged conditions at $26 \%$ AM depth (i.e., near the average psychometric threshold). Most points lie above the unity line, indicating a smaller SD of trial-by-trial firing rate during task engagement. This reduction was observed at all stimulus values averaged across all units in this subset (Fig. $4 A$ ). Comparison of AM depth values was conducted using a paired $t$ test with Bonferroni's adjusted $\alpha$ levels of $p=0.0014(0.01 /$ 7), all points of comparison $p<0.0014$ Since average firing rate did not change significantly during task engagement (Fig. $4 B$; none of the AM depth comparisons using a paired $t$ test approached Bonferroni's adjusted $\alpha$ levels), the improvement in classifier performance (Fig. $3 B$ ) and neurometric sensitivity (Fig. $3 A$ ) could be attributed largely to a decrease in response variability. We also calculated a baseline-corrected firing rate (see Materials and Methods), and still observed no significant changes in firing rate. These observations were further validated by calculating the coefficient of variation, which captures a firing-ratenormalized response variability (Fig. $4 C, C^{l}$ ). Coefficient of variation was significantly lower during task engagement at all stimulus values. All AM depth comparisons using a paired $t$ test reached Bonferroni's adjusted $\alpha$ levels of $p=0.0014(0.01 / 7)$.

The subpopulation of units for which classifier performance decreased or did not change during task engagement did not display a significant change in SD during task engagement, again using a paired $t$ test with Bonferroni's adjusted $\alpha$ levels (data not shown). This result suggests that the units with the greatest changes in response variability are driving the changes in classifier performance and neural sensitivity.

To examine the relationship between variability, firing rate, and neurometric sensitivity $\left(d_{F R}^{\prime}\right)$ for all units, we plotted each of the mentioned basic response properties against $d_{F R}^{\prime}$. We found that changes in variability from the engaged to disengaged condition had a significant negative correlation with changes in $d_{F R}^{\prime}$; as variability decreased, neural sensitivity increased (Fig. $5 \mathrm{~A}$; linear regression, $R^{2}=0.24, p<0.001$ ). However changes in firing rate had a near-zero correlation with $d_{F R}^{\prime}$ (Fig. $5 B$; linear regression, $\left.R^{2}=0.01, p<0.001\right)$. We next sought to demonstrate how changes in variability (Fig. $5 C$ ) could have influenced the population neurometric $d_{F R}^{\prime}$ functions (Fig. 3A). To explore the relative contributions of mean firing rate and response variability, we computed neurometric $d_{F R}^{\prime}$ functions based on simulated firing rate properties. Firing rate properties were quantified by fitting a normal distribution, truncated at zero, to each unit's firing rate at each stimulus value, and goodness of fit was assessed using a $\chi^{2}$ test. From this fitted distribution, an average firing rate and variance was determined for all units. To illustrate how neural $d_{F R}^{\prime}$ may have been altered by changes in the variance of the firing rate, we generated hypothetical neurometric $d_{F R}^{\prime}$ functions using the possible permutations of engaged or disengaged firing rate and engaged or disengaged SD (see Materials and Methods).

Using an engaged SD and firing rate yielded a simulated $d_{F R}^{\prime}$ that closely approximated the measured $d_{F R}^{\prime}$ (Fig. $5 C$ ), thereby confirming that the identical simulated parameters matched the 
measured data. We then tested how a disengaged firing rate and engaged response SD would affect $d_{F R}^{\prime}$. We found that the simulated $d_{F R}^{\prime}$ using a disengaged firing rate did not affect the shape of the neurometric function. This suggests that variability is the main driving factor in modulating the $d_{F R}^{\prime}$ neurometric functions. We also found that a simulated $d_{F R}^{\prime}$ that used the disengaged SD produced neurometric functions with low sensitivity regardless of which firing rate parameter was used (Fig. 5D). These simulated $d_{F R}^{\prime}$ neurometric functions demonstrate that variability is the main factor driving a change in the neurometric sensitivity.

\section{Decline in variability yields lower neural AM detection thresholds}

We next investigated how a decrease in response variability would affect subpopulation and individual unit $d_{F R}^{\prime}$ neurometric sensitivity, and its relation to psychometric thresholds (Fig. 1). A firing rate-based neural $d^{\prime}$ was recalculated for the two subpopulations of units parsed above (Fig. 3B). A neurometric function was obtained for each unit by plotting a firing rate-based $d_{F R}^{\prime}$ at each tested AM depth (see Materials and Methods). This was done separately for unit subpopulations in which classifier performance improved (Fig. 3B, blue circles) or declined (Fig. $3 B$, the subset of gray circles above the red circles) in the engaged condition relative to performance in the disengaged condition.

Average psychometric sensitivity was plotted alongside average neurometric sensitivity for units that displayed a decrease in variability (Fig. 6A) as well as for units that displayed no significant change in variability (Fig. $6 B$ ). The average neurometric function for units that decrease in variability more closely approximated the psychometric function. Furthermore, the average neurometric function for units that did not show significant change in variability more closely approximated the neurometric function obtained when animals were disengaged.

To investigate neural sensitivity, each unit's response function was fit with a sigmoidal function (see Materials and Methods), and neural AM detection threshold was obtained, where the fit $d_{F R}^{\prime}=1$. Figure $6 C$ insets show example fit neurometric functions. The cumulative distribution of neurometric thresholds for the two subpopulations is plotted in Figure $6 C$ (units that decrease variability, shown in Fig. $4 A$; units that displayed no change in variability), along with the range of psychometric performance (blue bar). A larger number of units that displayed decreased response variability had AM depth thresholds that more closely matched the perceptual thresholds than units that had no change in their response variability during task engagement. We then determined the percentage of units that displayed neurometric sensitivities greater than or equal to psychometric sensitivity. We found that $26 \%$ of all units had neural thresholds equal to or less than psychometric thresholds. Of these units $85 \%$ were units that displayed decreased response variability during task performance, and 15\% were units that showed no change in variability. This suggests that units
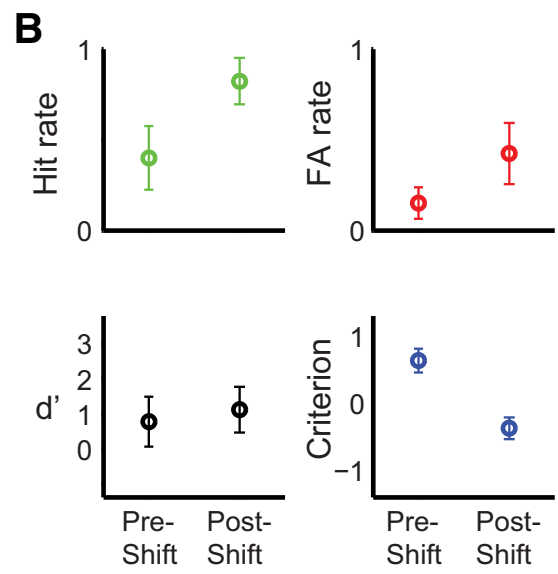

D

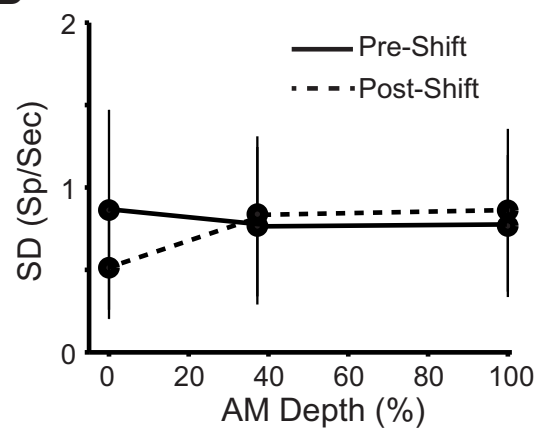

Figure 7. Change in response bias does not alter neural sensitivity. $\boldsymbol{A}$, Receiver operator characteristic isosensitivity curve (black showing predecision and postdecision bias relationship between $F A$ and hit rates (data points) during an example Errorbars represent binomial confidence intervals. $\boldsymbol{C}, \boldsymbol{D}$, Change in firing rate (FR, $\boldsymbol{C}$ ) and SD (D) from preshift (solid line) to postshift (dashed line) of response bias. Error bars represent SEM.

that decrease their response variability likely contribute to an animal's perceptual sensitivity to AM depth.

AM encoding remained stable when response bias was shifted Task engagement can have a significant effect on a unit's response to AM depth. Therefore, we asked whether neural encoding was robust to a change in response bias (i.e., a consistent willingness to report the presence or absence of a signal). According to SDT, an increase in response bias should not change psychometric sensitivity. Accordingly, we would not expect to observe a change in neural sensitivity. We measured responses to AM depth during task performance while inducing a change to an animal's response bias, in this case an increase in the willingness to report the presence of an AM modulation.

A within-session change in response bias was induced by increasing the probability of a Go trial being presented (Green and Swets, 1966). When the probability of a Go trial was increased, we observed an increase in hit rate and FA rate (Fig. $7 A, B$, top row). However, psychophysical $d^{\prime}$ did not change significantly (Fig. $7 B$, bottom left). Following an increase in Go trial probability, we observed an increase in response bias in 16 of 21 sessions. We used sessions in which a shift in response bias was observed to examine whether changes occurred to average trial-by-trial firing rate and SD. We found no differences in any of these response properties (Fig. 7C,D) when animals became more liberal in their bias. We also examined sessions where no shift was observed and did not find a significant difference between the first and last half of the session. We also observed no differences between shift and 
nonshift sessions. Therefore signal representation can remain stable when an animal changes its behavioral response.

\section{Discussion}

In an SDT framework, neural sensitivity depends on the variability of neural responses. In this study, we tested the hypothesis that neural response variability can decrease during task performance, thereby increasing neural sensitivity. We recorded telemetrically from the auditory cortex of freely moving gerbils as they performed a psychometric task. Our results suggest that task performance is associated with a net decrease in response variability: decreased variability in firing rate from trial to trial was the largest contributing factor to the observed increase of neural sensitivity. Moreover, units that displayed the greatest improvements in sensitivity had neural AM detection thresholds that were most closely aligned with simultaneously measured perceptual thresholds.

By comparing neural responses recorded when animals were performing a perceptual task with those recorded while animals were disengaged, we observed a decrease in variability that contributed significantly to the enhancement of neural sensitivity to AM depth (Fig. 3A). This increased neural sensitivity was driven by a subset of units (Fig. 6A) that showed a behaviorally gated decrease in response variability (Fig. $4 A, C$ ). Moreover, the psychometric thresholds for AM detection could best be explained by the subset of neural responses that displayed the greatest reduction in variability during task performance (Fig. 6C). Therefore, our results build on previous studies that show statedependent changes in variability, and provide evidence that a reduction in response variability is a plausible mechanism to improve perceptual ability on an AM detection task.

Since reducing response variability during task performance improves neural sensitivity and, plausibly, perceptual sensitivity, it is worth considering the underlying neural mechanisms. For example, researchers have shown that attention can modulate response properties via circuits involving the basal forebrain as well as prefrontal areas (Everitt and Robbins, 1997; Sarter et al., 2005). These pathways have also been found to have a causal role in modulating and enhancing sensory encoding (Goard and Dan, 2009; Fritz et al., 2010). Alternatively, circuits engaged during locomotion or arousal can also modulate auditory response properties (Otazu et al., 2009; McGinley et al., 2015a,b). In fact, any movement, including maintaining body posture, can modulate the auditory cortex (Schneider et al., 2014). Since firing rates were not found to be significantly different across conditions, motor activity is likely not influencing the observed changes in response properties.

According to SDT, an increase in response bias does not change psychometric sensitivity. Therefore, we assumed that neural sensitivity would also not be affected by response bias. Our observations were consistent with this model: neural sensitivity was stable as response bias changed (Fig. 6), suggesting that even though various aspects of task performance modulate neural response properties, once engaged in a task, sensory encoding can actually be quite stable. This finding also suggests that signals unrelated to sensory encoding (i.e., decision variables) do not influence neural sensitivity at the level of the primary auditory cortex.

This study emphasizes changes in variability, yet neural sensitivity also depends on response magnitude, which is also affected by behavioral state. Almost every aspect of a behavior, from locomotion to attention, can affect response magnitude and neural encoding. Task-related or state-related changes in response magnitude have been reported in the visual, olfactory, gustatory, and auditory systems. In the auditory system, both increases (Fritz et al., 2003, 2005; Lee and Middlebrooks, 2011; Niwa et al., 2012; Buran et al., 2014b) and decreases in response magnitude have been reported (Otazu et al., 2009; Schneider et al., 2014; Zhou et al., 2014; McGinley et al., 2015a). Therefore, behaviorally gated changes in response variability (present results) or magnitude (above) can each contribute to or detract from a subject's signal detection sensitivity. Reporting a metric that takes both response variability and magnitude into account could permit a better assessment of the relation between neural and perceptual sensitivity. It would be interesting to return to these studies to find how response variance and magnitude may act in concert to change neural sensitivity.

\section{References}

Bartlett EL, Wang X (2007) Neural representations of temporally modulated signals in the auditory thalamus of awake primates. J Neurophysiol 97:1005-1017. Medline

Billimoria CP, Kraus BJ, Narayan R, Maddox RK, Sen K (2008) Invariance and sensitivity to intensity in neural discrimination of natural sounds. J Neurosci 28:6304-6308. CrossRef Medline

Britten KH, Shadlen MN, Newsome WT, Movshon JA (1992) The analysis of visual motion: a comparison of neuronal and psychophysical performance. J Neurosci 12:4745-4765. Medline

Britten KH, Newsome WT, Shadlen MN, Celebrini S, Movshon JA (1996) A relationship between behavioral choice and the visual responses of neurons in macaque MT. Vis Neurosci 13:87-100. CrossRef Medline

Brückner G, Seeger G, Brauer K, Härtig W, Kacza J, Bigl V (1994) Cortical areas are revealed by distribution patterns of proteoglycan components and parvalbumin in the Mongolian gerbil and rat. Brain Res 658:67-86. CrossRef Medline

Buran BN, Sarro EC, Manno FA, Kang R, Caras ML, Sanes DH (2014a) A sensitive period for the impact of hearing loss on auditory perception. J Neurosci 34:2276-2284. CrossRef Medline

Buran BN, von Trapp G, Sanes DH (2014b) Behaviorally gated reduction of spontaneous discharge can improve detection thresholds in auditory cortex. J Neurosci 34:4076-4081. CrossRef Medline

Burton H, Sinclair RJ, Hong SY, Pruett JR Jr, Whang KC (1997) Tactilespatial and cross-modal attention effects in the second somatosensory and $7 \mathrm{~b}$ cortical areas of rhesus monkeys. Somatosens Mot Res 14:237267. CrossRef Medline

Caras ML, Sanes DH (2015) Sustained perceptual deficits from transient sensory deprivation. J Neurosci 35:10831-10842. CrossRef Medline

Carney LH, Ketterer AD, Abrams KS, Schwarz DM, Idrobo F (2013) Detection thresholds for amplitude modulations of tones in budgerigar, rabbit, and human. In: Basic aspects of hearing, pp 391-398. New York: Springer.

Chapman CE, Meftah el-M (2005) Independent controls of attentional influences in primary and secondary somatosensory cortex. J Neurophysiol 94:4094-4107. CrossRef Medline

Churchland MM, Yu BM, Cunningham JP, Sugrue LP, Cohen MR, Corrado GS, Newsome WT, Clark AM, Hosseini P, Scott BB, Bradley DC, Smith MA, Kohn A, Movshon JA, Armstrong KM, Moore T, Chang SW, Snyder LH, Lisberger SG, Priebe NJ, et al. (2010) Stimulus onset quenches neural variability: a widespread cortical phenomenon. Nat Neurosci 13:369378. CrossRef Medline

Cohen MR, Maunsell JH (2009) Attention improves performance primarily by reducing interneuronal correlations. Nat Neurosci 12:1594-1600. CrossRef Medline

Duda RO, Hart PE, Stork DG (2001) Pattern classification. NewYork: Wiley.

Ecker AS, Berens P, Cotton RJ, Subramaniyan M, Denfield GH, Cadwell CR, Smirnakis SM, Bethge M, Tolias AS (2014) State dependence of noise correlations in macaque primary visual cortex. Neuron 82:235-248. CrossRef Medline

Elhilali M, Fritz JB, Chi TS, Shamma SA (2007) Auditory cortical receptive fields: stable entities with plastic abilities. J Neurosci 27:10372-10382. CrossRef Medline

Elliott TM, Theunissen FE (2009) The modulation transfer function for speech intelligibility. PLoS Comput Biol 5:e1000302-e1000302. CrossRef Medline

Everitt BJ, Robbins TW (1997) Central cholinergic systems and cognition. Annu Rev Psychol 48:649-684. CrossRef Medline

Fee MS, Mitra PP, Kleinfeld D (1996) Automatic sorting of multiple unit 
neuronal signals in the presence of anisotropic and non-Gaussian variability. J Neurosci Methods 69:175-188. CrossRef Medline

Foley JM, Legge GE (1981) Contrast detection and near-threshold discrimination in human vision. Vis Res 21:1041-1053. CrossRef Medline

Fritz J, Shamma S, Elhilali M, Klein D (2003) Rapid task-related plasticity of spectrotemporal receptive fields in primary auditory cortex. Nat Neurosci 6:1216-1223. CrossRef Medline

Fritz J, Elhilali M, Shamma S (2005) Active listening: task-dependent plasticity of spectrotemporal receptive fields in primary auditory cortex. Hear Res 206:159-176. CrossRef Medline

Fritz JB, David SV, Radtke-Schuller S, Yin P, Shamma SA (2010) Adaptive, behaviorally gated, persistent encoding of task-relevant auditory information in ferret frontal cortex. Nat Neurosci 13:1011-1019. CrossRef Medline

Frund I, Haenel NV, Wichmann FA (2011) Inference for psychometric functions in the presence of nonstationary behavior. J Vis 11(6):pii:16. CrossRef Medline

Goard M, Dan Y (2009) Basal forebrain activation enhances cortical coding of natural scenes. Nat Neurosci 12:1444-1449. CrossRef Medline

Gold JI, Shadlen MN (2001) Neural computations that underlie decisions about sensory stimuli. Trends Cogn Sci 5:10-16. CrossRef Medline

Goris RL, Movshon JA, Simoncelli EP (2014) Partitioning neuronal variability. Nat Neurosci 17:858-865. CrossRef Medline

Green DM, Swets JA (1966) Signal detection theory and psychophysics. New York: Wiley.

Hill DN, Mehta SB, Kleinfeld D (2011) Quality metrics to accompany spike sorting of extracellular signals. J Neurosci 31:8699-8705. CrossRef Medline

Hubel DH, Henson CO, Rupert A, Galambos R (1959) Attention units in the auditory cortex. Science 129:1279-1280. CrossRef Medline

Johnson JS, Yin P, O'Connor KN, Sutter ML (2012) Ability of primary auditory cortical neurons to detect amplitude modulation with rate and temporal codes: neurometric analysis. J Neurophysiol 107:3325-3341. CrossRef Medline

Joris PX, Schreiner CE, Rees A (2004) Neural processing of amplitudemodulated sounds. Physiol Rev 84:541-577. CrossRef Medline

Lee CC, Middlebrooks JC (2011) Auditory cortex spatial sensitivity sharpens during task performance. Nat Neurosci 14:108-114. CrossRef Medline

Liang L, Lu T, Wang X (2002) Neural representations of sinusoidal amplitude and frequency modulations in the primary auditory cortex of awake primates. J Neurophysiol 87:2237-2261. Medline

Ludwig KA, Miriani RM, Langhals NB, Joseph MD, Anderson DJ, Kipke DR (2009) Using a common average reference to improve cortical neuron recordings from microelectrode arrays. J Neurophysiol 101:1679-1689. CrossRef Medline

Luna R, Hernández A, Brody CD, Romo R (2005) Neural codes for perceptual discrimination in primary somatosensory cortex. Nat Neurosci 8:1210-1219. CrossRef Medline

Machens CK, Schütze H, Franz A, Kolesnikova O, Stemmler MB, Ronacher B, Herz AV (2003) Single auditory neurons rapidly discriminate conspecific communication signals. Nat Neurosci 6:341-342. CrossRef Medline

Malone BJ, Scott BH, Semple MN (2007) Dynamic amplitude coding in the auditory cortex of awake rhesus macaques. J Neurophysiol 98:1451-1474. CrossRef Medline

McAdams CJ, Maunsell JH (1999) Effects of attention on the reliability of individual neurons in monkey visual cortex. Neuron 23:765-773. CrossRef Medline

McGinley MJ, David SV, McCormick DA (2015a) Cortical membrane potential signature of optimal states for sensory signal detection. Neuron 87:179-192. CrossRef Medline

McGinley MJ, Vinck M, Reimer J, Batista-Brito R, Zagha E, Cadwell CR, Tolias AS, Cardin JA, McCormick DA (2015b) Waking state: rapid variations modulate neural and behavioral responses. Neuron 87:1143-1161. CrossRef Medline

Narayan R, Graña G, Sen K (2006) Distinct time scales in cortical discrimination of natural sounds in songbirds. J Neurophysiol 96:252-258. CrossRef Medline

Niwa M, Johnson JS, O’Connor KN, Sutter ML (2012) Active engagement improves primary auditory cortical neurons' ability to discriminate temporal modulation. J Neurosci 32:9323-9334. CrossRef Medline

O'Connor KN, Johnson JS, Niwa M, Noriega NC, Marshall EA, Sutter ML (2011) Amplitude modulation detection as a function of modulation frequency and stimulus duration: comparisons between macaques and humans. Hear Res 277:37-43. CrossRef Medline

Otazu GH, Tai LH, Yang Y, Zador AM (2009) Engaging in an auditory task suppresses responses in auditory cortex. Nat Neurosci 12:646-654. CrossRef Medline

Parker AJ, Newsome WT (1998) Sense and the single neuron: probing the physiology of perception. Annu Rev Neurosci 21:227-277. CrossRef Medline

Quiroga RQ, Nadasdy Z, Ben-Shaul Y (2004) Unsupervised spike detection and sorting with wavelets and superparamagnetic clustering. Neural Comput 16:1661-1687. CrossRef Medline

Reynolds JH, Pasternak T, Desimone R (2000) Attention increases sensitivity of V4 neurons. Neuron 26:703-714. CrossRef Medline

Rosen MJ, Semple MN, Sanes DH (2010) Exploiting development to evaluate auditory encoding of amplitude modulation. J Neurosci 30:1550915520. CrossRef Medline

Rosen MJ, Sarro EC, Kelly JB, Sanes DH (2012) Diminished behavioral and neural sensitivity to sound modulation is associated with moderate developmental hearing loss. PloS One 7:e41514. CrossRef Medline

Rosen S (1992) Temporal information in speech: acoustic, auditory and linguistic aspects. Philos Trans R Soc Lond B Biol Sci 336:367-373. CrossRef Medline

Sarro EC, von Trapp G, Mowery TM, Kotak VC, Sanes DH (2015) Cortical synaptic inhibition declines during auditory learning. J Neurosci 35: 6318-6325. CrossRef Medline

Sarter M, Hasselmo ME, Bruno JP, Givens B (2005) Unraveling the attentional functions of cortical cholinergic inputs: interactions between signal-driven and cognitive modulation of signal detection. Brain Res Rev 48:98-111. CrossRef Medline

Schneider DM, Woolley SM (2010) Discrimination of communication vocalizations by single neurons and groups of neurons in the auditory midbrain. J Neurophysiol 103:3248-3265. CrossRef Medline

Schneider DM, Nelson A, Mooney R (2014) A synaptic and circuit basis for corollary discharge in the auditory cortex. Nature 513:189-194. CrossRef Medline

Singh NC, Theunissen FE (2003) Modulation spectra of natural sounds and ethological theories of auditory processing. J Acoust Soc Am 114:33943411. CrossRef Medline

Spitzer H, Desimone R, Moran J (1988) Increased attention enhances both behavioral and neuronal performance. Science 240:338-340. CrossRef Medline

Steinmetz PN, Roy A, Fitzgerald PJ, Hsiao SS, Johnson KO, Niebur E (2000) Attention modulates synchronized neuronal firing in primate somatosensory cortex. Nature 404:187-190. CrossRef Medline

Ter-Mikaelian M, Sanes DH, Semple MN (2007) Transformation of temporal properties between auditory midbrain and cortex in the awake Mongolian gerbil. J Neurosci 27:6091-6102. CrossRef Medline

Tolhurst DJ, Movshon JA, Dean AF (1983) The statistical reliability of signals in single neurons in cat and monkey visual cortex. Vis Res 23:775785. CrossRef Medline

Treue S, Maunsell JH (1999) Effects of attention on the processing of motion in macaque middle temporal and medial superior temporal visual cortical areas. J Neurosci 19:7591-7602. Medline

van Rossum MC (2001) A novel spike distance. Neural computation 13: 751-763. CrossRef Medline

Wang L, Narayan R, Graña G, Shamir M, Sen K (2007) Cortical discrimination of complex natural stimuli: can single neurons match behavior? J Neurosci 27:582-589. CrossRef Medline

Wang X, Lu T, Bendor D, Bartlett E (2008) Neural coding of temporal information in auditory thalamus and cortex. Neuroscience 157:484-494. CrossRef Medline

Yin P, Johnson JS, O'Connor KN, Sutter ML (2011) Coding of amplitude modulation in primary auditory cortex. J Neurophysiol 105:582-600. CrossRef Medline

Yoshida T, Katz DB (2011) Control of prestimulus activity related to improved sensory coding within a discrimination task. J Neurosci 31:41014112. CrossRef Medline

Zhou M, Liang F, Xiong XR, Li L, Li H, Xiao Z, Tao HW, Zhang LI (2014) Scaling down of balanced excitation and inhibition by active behavioral states in auditory cortex. Nat Neurosci 17:841-850. CrossRef Medline 